\title{
Bayesian Approach for Identification of Multiple Events in an Early Warning System
}

\author{
by Annie Liu and Masumi Yamada
}

\begin{abstract}
The 2011 Tohoku earthquake $\left(M_{\mathrm{w}} 9.0\right)$ was followed by a large number of aftershocks that resulted in 70 early warning messages in the first month after the mainshock. Of these warnings, a non-negligible fraction (63\%) were false warnings in which the largest expected seismic intensities were overestimated by at least two intensities or larger. These errors can be largely attributed to multiple concurrent aftershocks from distant origins that occur within a short period of time. Based on a Bayesian formulation that considers the possibility of having more than one event present at any given time, we propose a novel likelihood function suitable for classifying multiple concurrent earthquakes, which uses amplitude information. We use a sequential Monte Carlo heuristic whose complexity grows linearly with the number of events. We further provide a particle filter implementation and empirically verify its performance with the aftershock records after the Tohoku earthquake. The initial case studies suggest promising performance of this method in classifying multiple seismic events that occur closely in time.
\end{abstract}

\section{Introduction}

During the highly seismically active period after a major earthquake, multiple earthquakes can occur almost concurrently at different locations. In this case, the seismic waves measured by the ground sensors contain mixed signals from more than one source. If the detection algorithm assumes only one earthquake, the estimated earthquake parameters (e.g., location and magnitude) will not be accurate. These inaccurate estimates can lead to false warnings that are often observed after large earthquakes.

The 2011 Off the Pacific Coast of Tohoku Earthquake (hereafter called Tohoku earthquake) caused significant damage over a large area of northeastern Honshu. An earthquake early warning (EEW) was issued to the public in the Tohoku region about $8 \mathrm{~s}$ after the first $P$ arrival, which is $31 \mathrm{~s} \mathrm{after} \mathrm{the}$ origin time (Hoshiba and Iwakiri, 2011; Hoshiba et al., 2011; Sagiya et al., 2011). There was no blind zone, that is, warnings were received at all locations before the $S$ arrivals, because the earthquake was fairly far offshore.

The main earthquake was followed by a large number of aftershocks that resulted in 70 early warnings issued in the first month after the mainshock (Japan Meteorological Agency [JMA], 2011). Among these, 63\% of the warnings contained significant errors in which the estimated seismic intensities were at least two scales larger than the observed ones. As a comparison, only $29 \%$ of the warnings contained such errors prior to the Tohoku earthquake. Postevent analysis revealed that $73 \%$ of these errors could be attributed to failure to classify multiple concurrent earthquakes either from the same hypocenter separated by a short amount of time or from spatially distant origins (JMA, 2011). One of the primary reasons for these false alarms is that the current approach uses mainly $P$-wave arrival time to estimate the hypocenter.

In this paper, we propose a novel approach to detect and classify multiple concurrent earthquakes in the current JMA system framework. We introduce an approximate Bayesian method that estimates the location, magnitude, and origin time of multiple concurrent aftershocks. In contrast to the current JMA system, this approach produces multiple sets of estimation for earthquakes that occur closely in time. The experimental results from several case studies suggest that this approach can successfully detect and estimate the parameters of multiple concurrent earthquakes.

\section{Data and Processing}

This paper includes strong-motion data observed by the JMA seismic stations during and after the Tohoku earthquake. We evaluate the new classification approach on three sections of these records as summarized below. For each record, the values included in the JMA EEW are compared with the values that appear in the JMA catalog in Table 1.

\section{Data Set}

Case 1: 15 March 2011, 1:36:00-1:38:00 (Two Small Earthquakes). Early warnings were issued to the public based on 
Table 1

Summary of the Earthquake Information Studied in This Paper

\begin{tabular}{|c|c|c|c|c|c|c|c|c|c|c|}
\hline & $M_{\text {est }}$ & Longitude $\left({ }^{\circ}\right)$ & Latitude $\left({ }^{\circ}\right)$ & Depth $(\mathrm{km})$ & $\begin{array}{c}\text { Date } \\
(\mathrm{mm} / \mathrm{dd})\end{array}$ & $\begin{array}{c}\text { Time } \\
\text { (hh:mm:ss.ss) }\end{array}$ & $M$ & Longitude $\left({ }^{\circ}\right)$ & Latitude $\left({ }^{\circ}\right)$ & Depth $(\mathrm{km})$ \\
\hline \multirow[t]{2}{*}{ Case 1} & 5.9 & 138.6 & 36.9 & 10 & $03 / 15$ & 01:35:57.35 & 2.5 & 138.610 & 36.938 & 3.4 \\
\hline & & & & & $03 / 15$ & 01:36:12.72 & 3.3 & 139.879 & 35.526 & 20.5 \\
\hline \multirow[t]{2}{*}{ Case 2} & 7.6 & 142.1 & 38.2 & 30 & $03 / 20$ & $14: 19: 38.27$ & 3.0 & 141.935 & 38.286 & 42.3 \\
\hline & & & & & $03 / 20$ & 14:19:58.06 & 4.7 & 140.794 & 37.082 & 7.2 \\
\hline Case 3 & 8.6 & 142.7 & 38.2 & 10 & 03/11 & $14: 46: 48.08$ & 9.0 & 142.861 & 38.103 & 23.7 \\
\hline
\end{tabular}

The first four columns correspond to the real-time JMA EEW records. The last six columns are the values documented in the JMA unified hypocenter catalog. Both cases 1 and 2 contain two events. JMA magnitude is used for $M$.

an estimated JMA magnitude of 5.9 at $21 \mathrm{~s}$ after the first $P$-wave detection (see Data and Resources). However, the largest observed seismic intensity was only 2 in the JMA seismic intensity scale. As shown in Table 1, at least two events about $200 \mathrm{~km}$ apart and of magnitude 2.5 and 3.3 occurred within $15 \mathrm{~s}$. Because the second event started close in time to the wave arrivals of the first event, the EEW system treated these separate events as one single earthquake and, as a result, overestimated the magnitude.

Case 2: 20 March 2011, 14:19:00-14:21:00 (Two Small Earthquakes). The JMA EEW system estimated a magnitude of 7.6 at $6.6 \mathrm{~s}$ after the initial $P$-wave detection and issued a warning to the public (see Data and Resources). However, the largest observed seismic intensity was only 3. Again, as shown in Table 1, the overestimation can possibly be attributed to mistaking two smaller earthquakes about $150 \mathrm{~km}$ apart (JMA magnitude 3.0 and 4.7) that occurred within $5 \mathrm{~s}$ for one large earthquake, because the occurrence of the second event was close in time to the wave arrival of the first event.

Case 3: 11 March 2011, 14:46:00-14:49:00 (Tohoku Earthquake). To demonstrate that the method can also handle the classification of a single event, we also include the analysis of the Tohoku earthquake $\left(M_{\mathrm{w}} 9.0\right)$. An early warning was issued to the public in the Tohoku region about $8 \mathrm{~s}$ after the first $P$ arrival, which is $31 \mathrm{~s}$ after the origin time (see Data and Resources).

\section{Processing}

This paper uses the three-component acceleration data with a sampling rate of $100 \mathrm{~Hz}$ from about 200 stations. The acceleration data were first converted to Seismic Analysis Code format and decimated by a factor of 100 , reducing the sampling frequency to $1 \mathrm{~Hz}$. The decimation was not necessary but was used to reduce computation time. Each component of the decimated acceleration $k(t)$ was then converted to displacement $A(t)$. The conversion was done by twice integration of $k(t)$ using a recursive digital filter with the frequency response of a mechanical seismometer (Katsumata, 2008).

$$
\begin{aligned}
A(t)= & g_{0} \times\left[k(t)+h_{0} \times k(t-1)+h_{1} \times k(t-2)\right]-h_{2} \\
& \times A(t-1)-h_{3} \times A(t-2),
\end{aligned}
$$

in which the function gain factor $g_{0}$ and filter constants $h_{0}, h_{1}$, $h_{2}$, and $h_{3}$ depend on the sampling frequency, damping constant, and natural period of the seismometer. For a JMA seismometer with $100 \mathrm{~Hz}$ sampling, 0.55 damping constant, and $6 \mathrm{~s}$ natural period, the values correspond to

$g_{0}=0.0000248691025, \quad h_{0}=1.0, \quad h_{1}=1.0$,

$h_{2}=-1.9889474, \quad h_{3}=0.9895828$.

The following approach to classification uses both the vector sum of the three component displacement $A(t)$ as well as the vertical component of acceleration $k(t)$. The picking is done with short-term average/long-term average of $k(t)$ with a short-term window of $1 \mathrm{~s}$ and long-term window of $10 \mathrm{~s}$. The method also computes expected $P$ - and $S$-wave arrival times $\left(t_{P}\right.$ and $\left.t_{S}\right)$ to determine whether a station should have observed a $P$ wave, $S$ wave, or neither. These arrival times are computed with the JMA 1D layered velocity structure (Ueno et al., 2002).

\section{Bayesian Method}

The problem of continuous parameter estimation for multiple events can be formulated as a Bayesian inference problem. Let $\theta$ be the vector of parameters that characterizes an event and $\Theta$ be a set of events that are parametrized by $\theta$, such that $\Theta=\left\{\phi,\left\{\theta_{1}\right\}, \ldots,\left\{\theta_{1}, \theta_{2}, \ldots\right\}\right\}$. Suppose $\mathbf{z}_{1: t}$ is the complete history of observations from all the stations until the current time $t$, the posterior $P\left(\Theta_{t} \mid \mathbf{z}_{1: t}\right)$ reveals the distribution of information of current ongoing events at time $t$ given the evidence and prior information.

$$
P\left(\Theta_{t} \mid \mathbf{z}_{1: t}\right)=\frac{P\left(\mathbf{z}_{t} \mid \Theta_{t}\right) P\left(\Theta_{t} \mid \mathbf{z}_{1: t-1}\right)}{P\left(\mathbf{z}_{t} \mid \mathbf{z}_{1: t-1}\right)},
$$

in which $P\left(\mathbf{z}_{t} \mid \Theta_{t}\right)$ is the likelihood function and is typically denoted as $L, L\left(\mathbf{z}_{t} \mid \Theta_{t}\right)=P\left(\mathbf{z}_{t} \mid \Theta_{t}\right) . P\left(\Theta_{t} \mid \mathbf{z}_{1: t-1}\right)$ is the updated prior at time $t$,

$$
P\left(\Theta_{t} \mid \mathbf{z}_{1: t-1}\right)=\int P\left(\Theta_{t} \mid \Theta_{t-1}\right) P\left(\Theta_{t-1} \mid \mathbf{z}_{1: t-1}\right) \mathrm{d} \Theta_{t-1},
$$

and $P\left(\Theta_{0} \mid \mathbf{z}_{0}\right) \equiv P\left(\Theta_{0}\right)$ is the prior distribution of $\Theta$. 


\section{Particle Filter}

In general, equation (3) does not have a closed-form solution, and there are several suboptimal solutions to approximate the posterior distribution (Arulampalam et al., 2002), one of which is grid search. Grid search, though simple to implement, suffers a few problems. First of all, when the parameters are continuous and not sufficiently restricted, the method cannot cover the complete parameter space because there can only be a finite number of grids. Second, the grid size is predefined; and, as a result, it requires a large number of grids to achieve good coverage at a desired resolution.

Another solution is the particle filter (PF), which is a sequential Monte Carlo method that approximates the posterior distribution with a set of weighted particles (Doucet et al., 2001). As the number of particles goes to infinity, the solution from PF approaches the optimal solution. There is a rich literature on $\mathrm{PF}$ and its variation (Liu and Chen, 1998; Doucet et al., 2001; Arulampalam et al., 2002). The basic procedure is summarized below for reference.

Sampling. At the beginning of each iteration, the value of each particle is drawn from an important density function $q\left(\Theta_{t}^{i} \mid \Theta_{t-1}^{i}, \mathbf{z}_{t}\right)$. For $i=1, \ldots, N$

$$
\Theta_{t}^{i} \sim q\left(\Theta_{t}^{i} \mid \Theta_{t-1}^{i}, \mathbf{z}_{t}\right),
$$

in which $\sim$ denotes that the sample $\Theta_{t}^{i}$ is drawn according to the distribution $q(\cdot)$.

Weight Update. PF approximates the posterior with a collection of weighted particles:

$$
P\left(\Theta_{t} \mid \mathbf{z}_{1: t}\right) \approx \sum_{i=1}^{N} w_{t}^{i} \times \delta\left(\Theta_{t}-\Theta_{t}^{i}\right),
$$

in which $w_{t}^{i}$ is the weight for the particle $i$ at time $t$. The sum of total weights are normalized to 1 .

$$
\sum_{i=1}^{N} w_{t}^{i}=1
$$

The weights for all particles are updated as new evidence $\mathbf{z}_{t}$ comes in and renormalized at the end of each update:

$$
w_{t}^{i} \propto w_{t-1}^{i} \frac{L\left(\mathbf{z}_{t} \mid \Theta_{t}^{i}\right) P\left(\Theta_{t}^{i} \mid \Theta_{t-1}^{i}\right)}{q\left(\Theta_{t}^{i} \mid \Theta_{t-1}^{i}, \mathbf{z}_{t}\right)},
$$

in which $q(\cdot)$ is the same important density that appears in the sampling step. To simplify the calculation, $q(\cdot)$ is often chosen to be the transition prior $P\left(\Theta_{t}^{i} \mid \Theta_{t-1}^{i}\right)$. Because the terms cancel out in the right side, the new weight is directly proportional to the likelihood $L\left(\mathbf{z}_{t} \mid \Theta_{t}^{i}\right)$.

Resampling. Because the posterior is approximated with discrete particles, the system suffers sample degeneracy after a few update iterations when the weight is concentrated on a very small number of particles. The decrease in weight variance determines the degree of degeneracy that can be approximated with $\widehat{N_{\text {eff }}}$ (Arulampalam et al., 2002),

$$
\widehat{N_{\mathrm{eff}}}=\frac{1}{\sum_{i=1}^{N}\left(w_{t}^{i}\right)^{2}} \text {. }
$$

Small $\widehat{N_{\text {eff }}}$ indicates severe degeneracy in which case resampling is required. Resampling essentially eliminates particles with negligible weight by generating a new set of $N$ equally weighted particles according to current distribution $P\left(\Theta_{t} \mid \mathbf{z}_{1: t}\right)$. There exists many methods for sampling from a discrete distribution, which we will not discuss here.

Each iteration typically involves one sampling and one weight update. Resampling only happens when $\widehat{N_{\text {eff }}}$ drops below a certain threshold.

\section{Model}

In the rest of the section, we discuss the practical implementation details of a PF-based real-time parameter estimation system for multiple earthquakes. The parameters we would like to estimate are $\theta=\left[x, y, D, M, t_{0}\right]$, in which $x$ is longitude in degrees, $y$ is latitude in degrees, $D$ is depth in kilometers, $M$ is JMA magnitude, and $t_{0}$ is origin time. The complete pseudocode (algorithm 1 in Fig. A1) is included in the Appendix.

Prior Distribution. The prior $P(\theta)$ determines how the particles are initialized. A good prior encodes geographical information such as the location of nearby fault lines to the station that first triggered and the most common magnitudes generated at the fault lines. This information can be compiled from historical earthquake catalog for each station and used in real time when initializing the PF. If prior information is absent, then a flat prior can be used instead. The choice of prior distribution affects the quality of the estimates and the convergence rate. Prior distribution of large coverage may cause the initial estimates to be unstable because little evidence is present. Priors of small coverage may result in slow convergence or false convergence (converging at the wrong values). These trade-offs can be evaluated empirically. In this paper, we use a uniform flat prior of $\pm 100 \mathrm{~km}$ for location, $\pm 10 \mathrm{~km}$ for depth, \pm 1 magnitude for event magnitude, and $\pm 10 \mathrm{~s}$ for event origin time.

Likelihood Function. The performance of the PF for parameter estimation depends largely upon the design of the likelihood function. In addition to the arrival time and measured amplitude from the triggered stations that current JMA approach uses, our likelihood function also utilize the same information from nontriggered stations as well because they also convey important information about the event.

In this paper, we use the attenuation relationship developed by JMA for magnitude estimation. The relationship is stated as follows (Hoshiba and Ozaki, 2013). Let $A_{\max }$ be the maximum displacement measured by a seismometer after the onset of an 
(a)

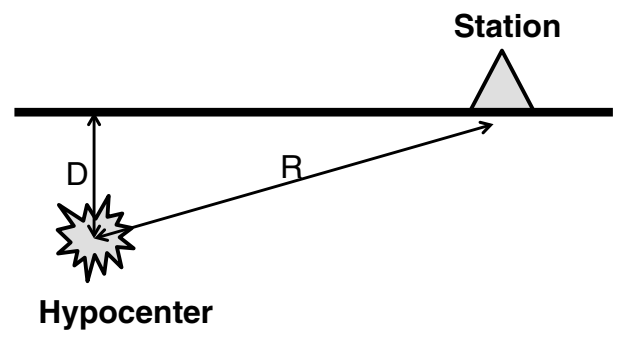

(b)

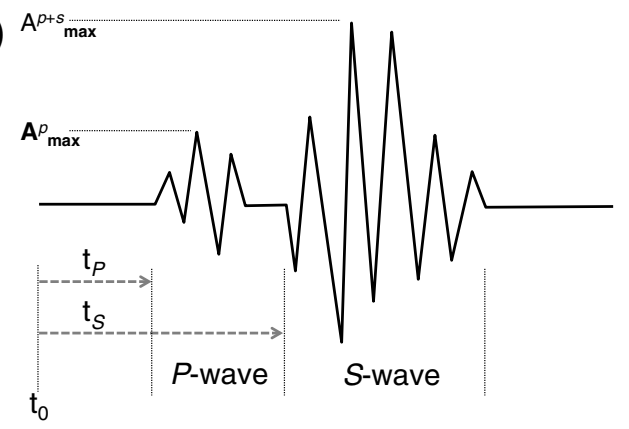

Figure 1. Illustrations of the parameters used in the Model section. (a) Hypocenter and seismic station and (b) amplitude and arrival times. $t_{P}$ and $t_{S}$ mark the arrival time of the $P$ wave and $S$ wave since the start of the earthquake at $t_{0}, t_{P} \leq t_{S}$.

event. The earthquake $P$-wave and $S$-wave magnitudes $M_{P}$ and $M_{S}$ can be expressed as a function of the linear distance from the station to the hypocenter $(R)$, the depth of the hypocenter $(D)$, and the maximum displacement for $P$ wave $\left(A_{\max }^{P}\right)$, or the maximum displacement of the entire duration $\left(A_{\max }^{P+S}\right)$ :

$$
\begin{aligned}
0.72 M_{P}= & \log A_{\max }^{P}+1.2 \log R+5 \times 10^{-4} R-5.0 \\
& \times 10^{-3} D+0.46, \\
0.87 M_{S}= & \log A_{\max }^{P+S}+\log R \\
& +1.9 \times 10^{-3} R-5.0 \times 10^{-3} D+0.98 .
\end{aligned}
$$

The relationship between the parameters is illustrated in Figure 1. These formulas are specifically tailored for the geologic compositions in Japan (see Data and Resources). The $P$-wave and $S$-wave magnitudes are expressed in terms of the maximum displacement $A_{\max }$ rather than the maximum acceleration or velocity because the scatter of displacement is smaller.

Given equations (10) and (11) and that the displacement is lognormally distributed $A \sim \ln \mathcal{N}\left(\mu, \sigma^{2}\right)$, we propose the following likelihood function for a single station:

$$
L\left(\mathbf{z} \mid x, y, D, M, t_{0}\right)=\frac{\exp \frac{-\left(\log A_{\max }-\log A_{\text {exp }}\right)^{2}}{2 \sigma^{2}}}{A_{\max } \times \sigma \sqrt{2 \pi}} .
$$

Here $A_{\text {exp }}$ is the expected $A_{\text {max }}$ and $\sigma$ is the standard deviation of displacement measurement. Depending on whether the station has observed $P$ wave, $S$ wave, or neither, the expected maximum displacement and its standard deviation are different. For convenience, by rearranging equations (10) and (11), we can compute $A_{\exp }$ and $\sigma$ for the following three cases.

Note that equation (12) is based on an amplitude that departs from standard arrival-time-based methods. The main reason for adopting this approach is the observation that the information of no shaking is critical in separating and classifying multiple earthquakes that occur close in space and time. This will be further discussed in the Discussion section.

- Has not observed any seismic wave:

$$
\log A_{\exp }=\log A_{\text {noise }}, \quad \sigma=\sigma_{\text {noise }} .
$$

- Has observed $P$ wave:

$$
\begin{aligned}
\log A_{\exp }= & 0.72 M_{P}-1.2 \log R-5 \times 10^{-4} R+5.0 \\
& \times 10^{-3} D-0.46, \quad \sigma=\sigma_{P} .
\end{aligned}
$$

- Has observed $S$ wave:

$$
\begin{aligned}
\log A_{\exp }= & 0.87 M_{S}-\log R-1.9 \times 10^{-3} R+5.0 \\
& \times 10^{-3} D-0.98, \quad \sigma=\sigma_{S} .
\end{aligned}
$$

$A_{\text {noise }}$ and $\sigma_{\text {noise }}$ are the noise in displacement measurement due to recent environmental noise and can be computed independently for each station by keeping a running window. $\sigma_{P}$ and $\sigma_{S}$ can be precomputed from historical earthquake data. The decision of which $A_{\exp }$ to compute for a station depends on whether $P$ wave, $S$ wave, or neither has arrived at the station. The expected travel time of $P$ wave and $S$ wave $\left(t_{P}\right.$ and $\left.t_{S}\right)$ can be computed with ray theory, given the relative location of the station to a hypocenter $(x, y, D)$. Comparison between $t_{P}, t_{S}$, the absolute current time $t$, and the absolute event start time $t_{0}$ gives direct estimation of which $A_{\text {exp }}$ to compute for a station. Figure 2 provides an illustrative summary of these design ideas.

This design of the likelihood function is based on the maximum displacement $A_{\max }$ that a seismometer observes during the shaking of a $P$ or $S$ wave. However, a seismometer may not observe the maximum displacement immediately after the wave arrival. In this case, the initial estimates can be highly incorrect using this likelihood function. A simple delay function $g(\cdot)$ can be included to approximate the instantaneous displacement before the maximum is observed,

$$
A_{\exp }=g\left(t-t_{0}-t_{P}\right) A_{\max }, \quad 0 \leq g(\cdot) \leq 1,
$$

in which $t$ and $t_{0}$ are the absolute current time and the absolute event origin time, respectively. $t_{P}$ is the expected $P$-wave travel time. An example of $g(t)$ is a left shifted sigmoid function.

The likelihood $L(\cdot \mid \cdot)$ is applied in each time step to update the weight of each particle. Assuming that each station makes independent observations and the collection of observations from all stations is $\mathbf{z}$, the complete likelihood function becomes 


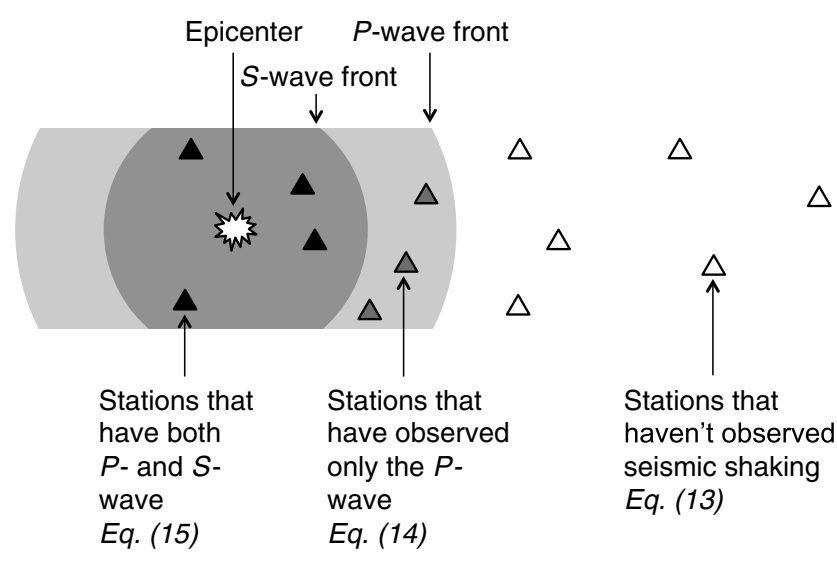

Figure 2. Illustrative summary of the design of a single-station likelihood function. The expected observation made by a station depends on whether it should have observed a $P$ wave, $S$ wave, or neither, given a hypocenter estimate.

$$
L\left(\mathbf{z} \mid x, y, D, M, t_{0}\right)=\prod_{i=1}^{n} L\left(z_{i} \mid x, y, D, M, t_{0}\right),
$$

in which $n$ is the number of stations. The independence assumption is a minor simplification because nearby stations may have correlated observations.

\section{Generalized Particle Filter}

Particles are initialized according to a prior distribution on the parameters. Because we are approximating an unbounded and continuous 5D space with a bounded and discrete one, care must be taken to ensure the particles have sufficient coverage and the number of required particles stays bounded. This is especially important for the seismic application because both the number of parameters and the range of values they can take are large. One way to ensure particle diversity with a limited number of particles is to adopt the regularized PF (RPF) approach (Arulampalam et al., 2002).

RPF differs from common PF only in the resampling stage. Rather than sampling from a discrete approximation of the posterior density $P(\cdot \mid \mathbf{z})$ as in equation (6), RPF samples from a continuous approximation (Musso et al., 2001). More specifically, RPF draws samples from the approximation,

$$
P(\theta \mid \mathbf{z}) \approx \sum_{i=1}^{N} w_{i} \times K_{h}\left(\theta-\theta_{i}\right)
$$

in which $K_{h}(\theta)=\frac{1}{h} K(\theta / h), h>0$ is the rescaled kernel density of $K(\cdot), h$ is the bandwidth, and $w_{i}$ is the normalized weight for the particle $i$. As a comparison, $K_{h}(\theta)$ is the Dirac delta function $\delta(\theta)$ in the regular PF. Special care is given to the design of kernels to minimize the error between the approximated and actual distribution. Under the assumption that all particles are equally weighted and the density is Gaussian, the optimal kernel is the Epanechnikov kernel (Musso et al., 2001).

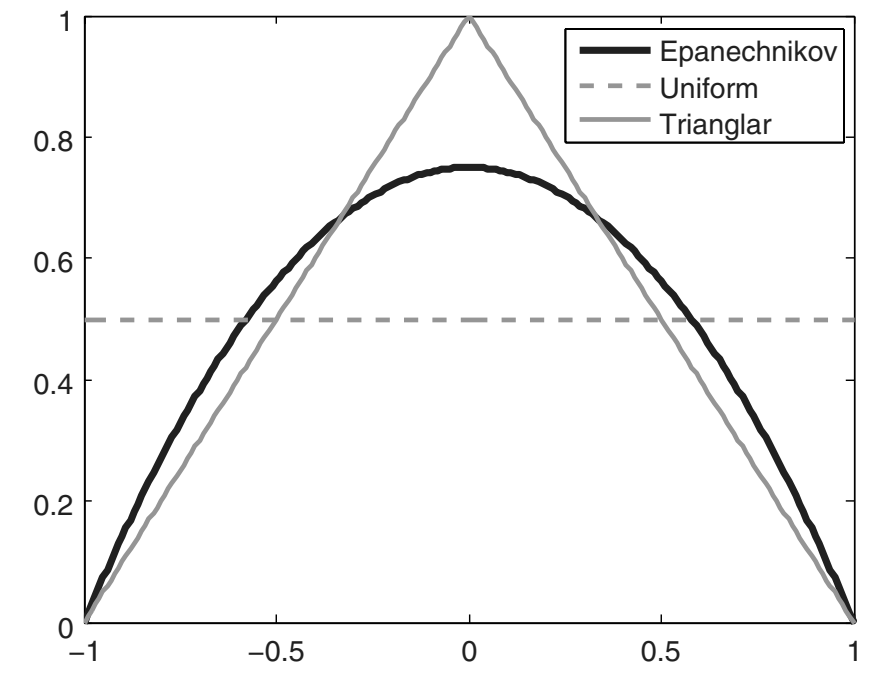

Figure 3. Some popular smoothing kernels used in regularized particle filter (RPF). Each kernel integrates to 1 to ensure that the resulting density is still a probability density function.

$$
K_{\mathrm{opt}}(x)= \begin{cases}\frac{n_{x}+2}{2 C_{n_{x}}}(1-\|x\|) & \|x\|<1 \\ 0 & \text { otherwise }\end{cases}
$$

in which $n_{x}$ is the dimension of the parameter space, $C_{n_{x}}$ is the volume of the unit hypersphere in $\mathbb{R}^{n_{x}}$. Figure 3 lists a few popular kernels in the literature.

The bandwidth vector $h$ can be chosen proportionally to the variance in the particle population by computing the Cholesky decomposition of the empirical covariance matrix (Bickel and Levina, 2008).

\section{Approximate Method for Multiple Concurrent Earthquakes}

$\mathrm{PF}$ allows for solving the Bayesian inference problem when exact inference is intractable; however, for the estimates to approach the optimal solution, the number of required particles must grow exponentially with the number of events.

Fortunately, as shown in historical records, the probability of having $n$ concurrent earthquakes within a time window of $60 \mathrm{~s}$ is exponentially small for large $n(n>3)$. Incorporating this information into the prior distribution can significantly reduce the size of the state space. However, the state space may still be too large for efficient real-time computation even with this information. For example, suppose the earthquake can be parameterized by a five-parameter vector $\theta, \theta=\left[x y D M t_{0}\right]^{T}$, in which $[x y D]^{T}$ is the [longitude, latitude, depth] coordinate, $M$ is the event magnitude, and $t_{0}$ is the event starting time. In the presence of $n=3$ earthquakes, the states to be searched reside in a $5 \times 3=15$ dimensional space.

This amount of computation may be executable in reasonable time on a supercomputer or a networked system of computers with parallel implementation of PF (Miao et al., 2010; Durham and Geweke, 2013). In this paper, however, 
we propose a simple heuristic to keep track of multiple earthquakes. The heuristic has the desired property such that the complexity grows linearly with the number of the events.

As a first approximation, the heuristic initializes separate PFs $\mathrm{pf}_{1}\left(\theta_{1}\right), \mathrm{pf}_{2}\left(\theta_{2}\right), \ldots$ for all possible earthquakes rather than keeping track of all events within one $\mathrm{PF}$ $\operatorname{pf}\left(\Theta=\left\{\theta_{1}, \theta_{2}, \ldots\right\}\right)$. Each PF communicates its current estimate $\hat{\theta}$ at the end of each update step to all other PFs. Specifically, each $\mathrm{PF}\left(\mathrm{pf}_{i}\right)$ computes the following posterior at time $t$,

$$
P\left(\theta_{i}^{t} \mid \mathbf{z},\left\{\hat{\theta}_{j}^{t-1}, j \neq i\right\}\right) .
$$

This approximation breaks down the $5 n$ state space in which $n$ is the number of concurrent earthquakes. It dramatically reduces the required computations to keep all events estimation up to date; however, it is suboptimal because all the particles from $\mathrm{pf}_{1}, \mathrm{pf}_{2}, \ldots$ combined only cover a small fraction of the complete parameter space.

The heuristic initializes a new PF with each single station $P$-wave pick, using a high threshold such that noisy detections are filtered out. Because local detection can be due to an existing event that is being tracked by another PF, it is necessary to condition new initialization on a separate metric. A natural choice of metric is $\mathbb{P}\left[z \mid \hat{\theta}_{1}, \hat{\theta}_{2}, \ldots\right]$, that is, the probability that the triggered measurement can be explained by existing events. Computation of this metric can follow directly from the single-station likelihood calculation as in equation (12); however, determining $A_{\exp }$ is nontrivial in this case because it involves computing the additive effect of the interference of multiple wavefronts. We propose an alternative metric that allows for rapid computation; the metric is the probability of shaking due to any of the existing events and threshold on the highest probability:

$$
\max _{i} \mathbb{P}\left[z \mid \hat{\theta}_{i}\right]=\max _{i} L\left(z \mid \hat{\theta}_{i}\right)\left\{\begin{array}{cl}
<\tau, & \text { initialize new pf } \\
\geq \tau, & \text { do nothing }
\end{array} .\right.
$$

By tuning the threshold $\tau$, we adjust how conservative the system is in declaring new events. The complete algorithm is outlined in Figure A1 for reference.

\section{Results}

We carried out the PF parameter estimation approach on the data described in the Data and Processing section, using a flat prior around the first triggered station and 1000 particles for each PF. The algorithm updates at a $1 \mathrm{~s}$ interval, and all experiments were run in simulated real time.

Case 1: 15 March 2011, 1:36:00-1:38:00 (Two Small Earthquakes)

Twenty trials were performed during this period of time. Snapshots of the particle distribution for one of the runs are shown in Figure 4. The averaged time histories of the estimated parameters across all 20 runs were compared against the JMA unified catalog (marked as dotted lines) in Figure 5.
The standard deviations across all runs are included as the error bars. The labeled $x$ axis corresponds to seconds because of the first detection of the first event. As the results demonstrate, the first PF was initiated at the first $P$-wave arrivals, and $15 \mathrm{~s}$ later, another PF was created. This approach successfully identified the two separate events. In addition, all estimates converge within $10 \mathrm{~s}$ after the initializations. On average, the method is able to localize the epicenters to within $20 \mathrm{~km}$ and produce magnitude estimates with an error of \pm 1 , relative to the JMA unified hypocenter catalog (Table 1).

Case 2: 20 March 2011, 14:19:00-14:21:00 (Two

Small Earthquakes)

We repeated the analyses for the dataset of case 2, in which two small earthquakes occurred $5 \mathrm{~s}$ apart. The snapshots of particle distributions and time series of estimated parameters are included in Figures 6 and 7. In this example, because the first event occurred offshore and there were fewer near-source recordings, localization and estimation of other parameters are more challenging than for case 1 . Indeed, the results showed that the estimates converge slower (about $30 \mathrm{~s}$ for event $\mathrm{A}$ ), and the averaged localization error was relatively large (about $80 \mathrm{~km}$ for event A), relative to the JMA unified hypocenter catalog (Table 1). However, the algorithm was still able to identify and separate the two events and to provide accurate estimates of their magnitudes to within \pm 0.5 .

Case 3: 11 March 2011, 14:46:00-14:49:00 (Tohoku

Earthquake)

We used the dataset of the Tohoku earthquake to show that the approach also works for a single event. The snapshots of particle distributions and time series of estimated parameters are included in Figures 8 and 9. Because the event was originated offshore, there was substantial localization error in the initial estimates. However, the averaged error decreased with time and converged at less than $40 \mathrm{~km}$ at $40 \mathrm{~s}$ after the initial $P$ wave arrival. The magnitude estimate grew from 6.0 to 8.4 as the earthquake rupture propagated, which is consistent with the earthquake rupture physics. At convergence, all five estimated parameters were close to the values in the JMA catalog.

\section{Discussion}

Current JMA methods to detect and associate multiple earthquakes perform well when the events are far apart in space or time. However, they have been shown to generate many false alarms when events are close in space or time (Sagiya et al., 2011). The empirical studies suggest the particle-based heuristic can successfully separate multiple concurrent seismic events and provide reasonable estimates of their parameters. Also, the speed of convergence may be improved by incorporating $P$-wave arrival time in the likelihood, that is, the residual between observed and predicted $P$-wave arrival times. The results show that estimated parameters converge in less than $10 \mathrm{~s}$ for inland earthquakes. For 
(a)

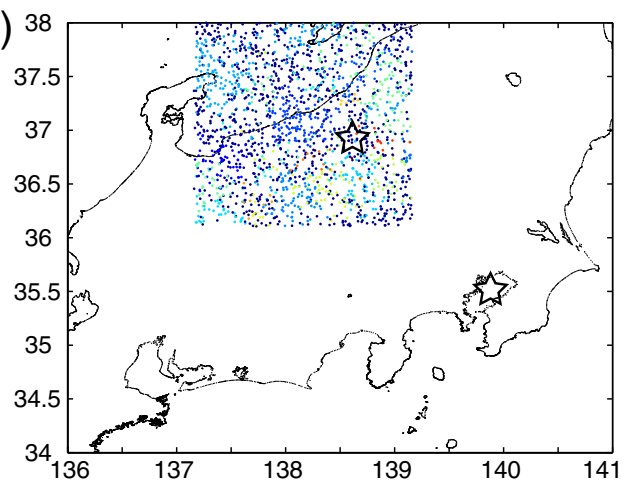

(c) 38

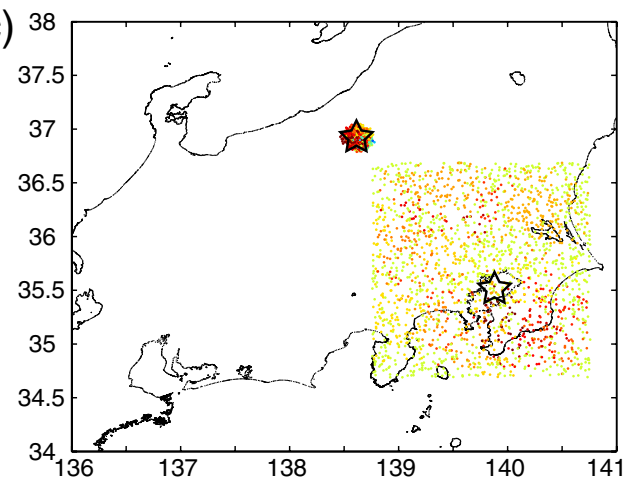

(b) 38

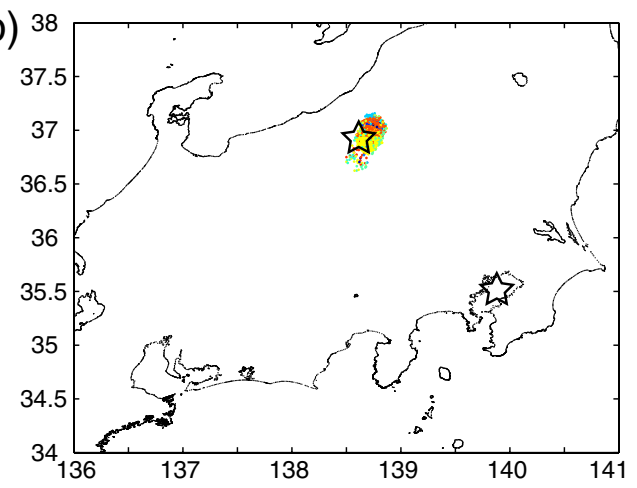

(d)

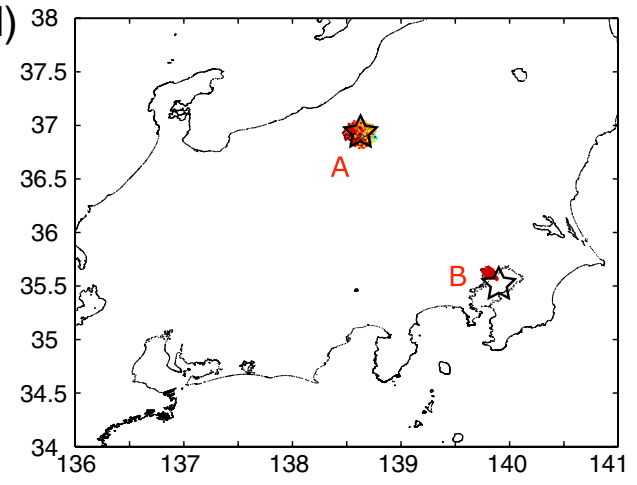

Figure 4. Distributions of 2000 particles visualized on the map at (a) $1 \mathrm{~s}$, (b) $2 \mathrm{~s}$, (c) $14 \mathrm{~s}$, and (d) $17 \mathrm{~s}$ after 01:36:07 on 15 March 2011. The time corresponds to seconds elapsed since the first $P$-wave detection. The official epicenters for the two events as they appeared in the JMA catalog are marked as stars and labeled in (d) for reference. The color version of this figure is available only in the electronic edition.
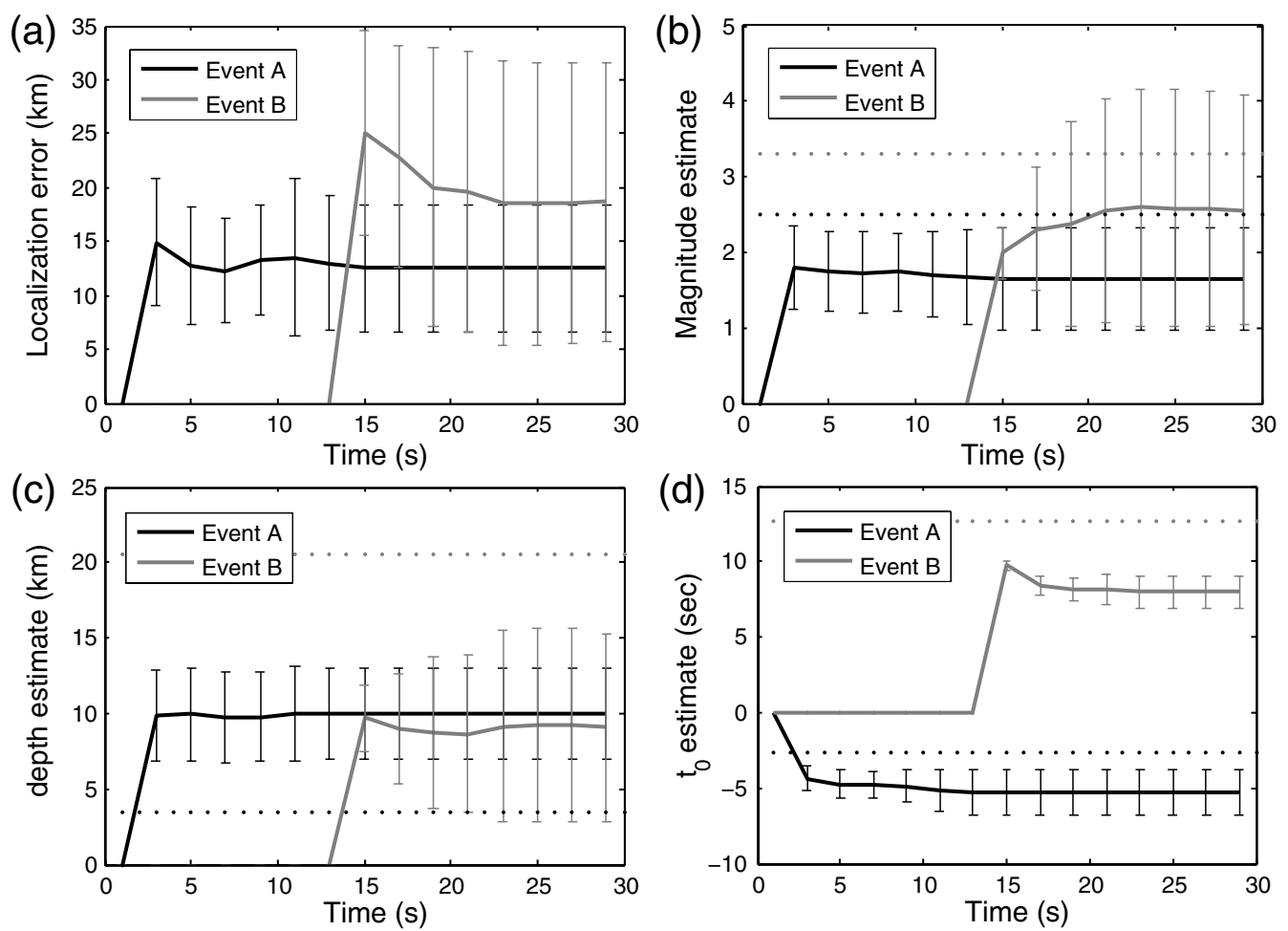

Figure 5. Results compiled from 20 independent runs for the period between 01:36:07 and 01:36:37 on 15 March 2011. Time histories of (a) the localization error, (b) magnitude, (c) depth of the hypocenter, and (d) origin time of the event. The two events are labeled according to Figure 4d. Averaged time histories across all 20 runs are marked as solid lines, and the official values that appear in the JMA catalog are marked as dashed lines. The standard deviations across all runs are shown as error bars. The time displayed on the $x$ axis is relative to the first pick from the earliest event. 
(a)

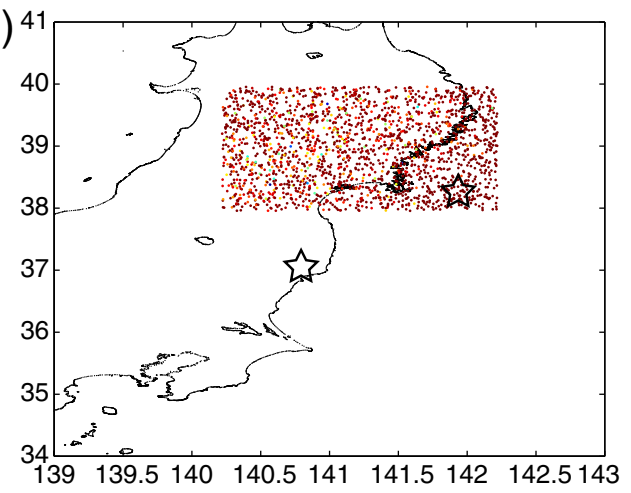

(c) 4

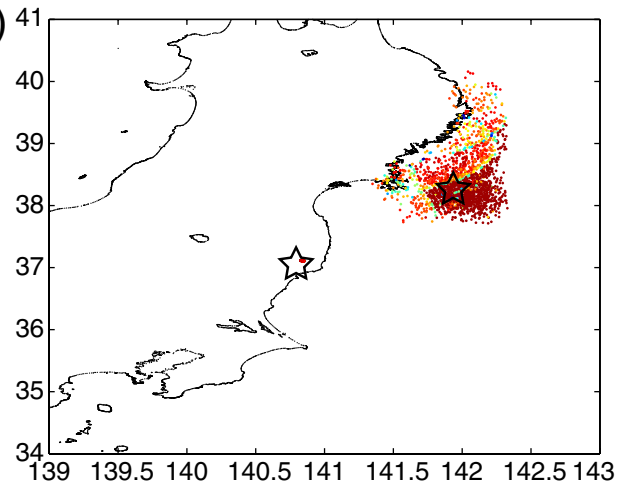

(b)

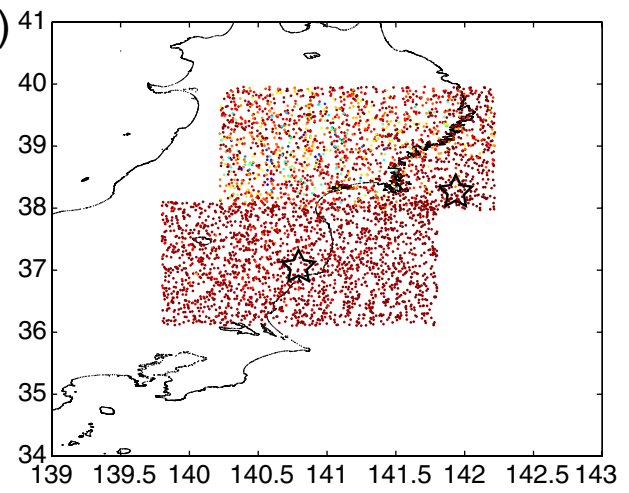

(d) ${ }^{4}$

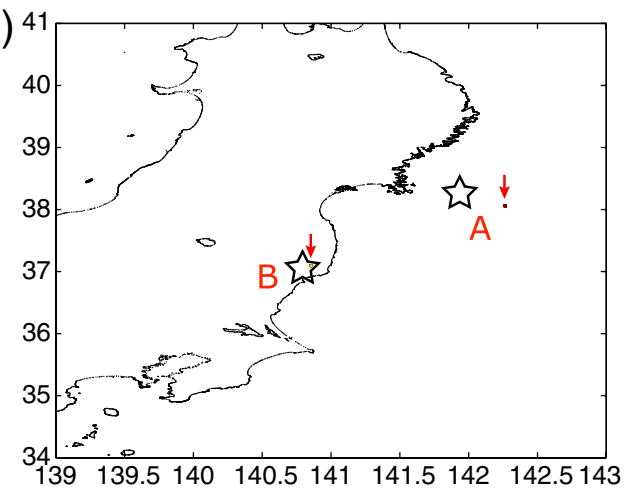

Figure 6. Distributions of 2000 particles visualized on the map at (a) $2 \mathrm{~s}$, (b) $7 \mathrm{~s}$, (c) $17 \mathrm{~s}$, and (d) $37 \mathrm{~s}$ after 14:19:56 on 20 March 2011. The symbols are defined as in Figure 4. The color version of this figure is available only in the electronic edition.
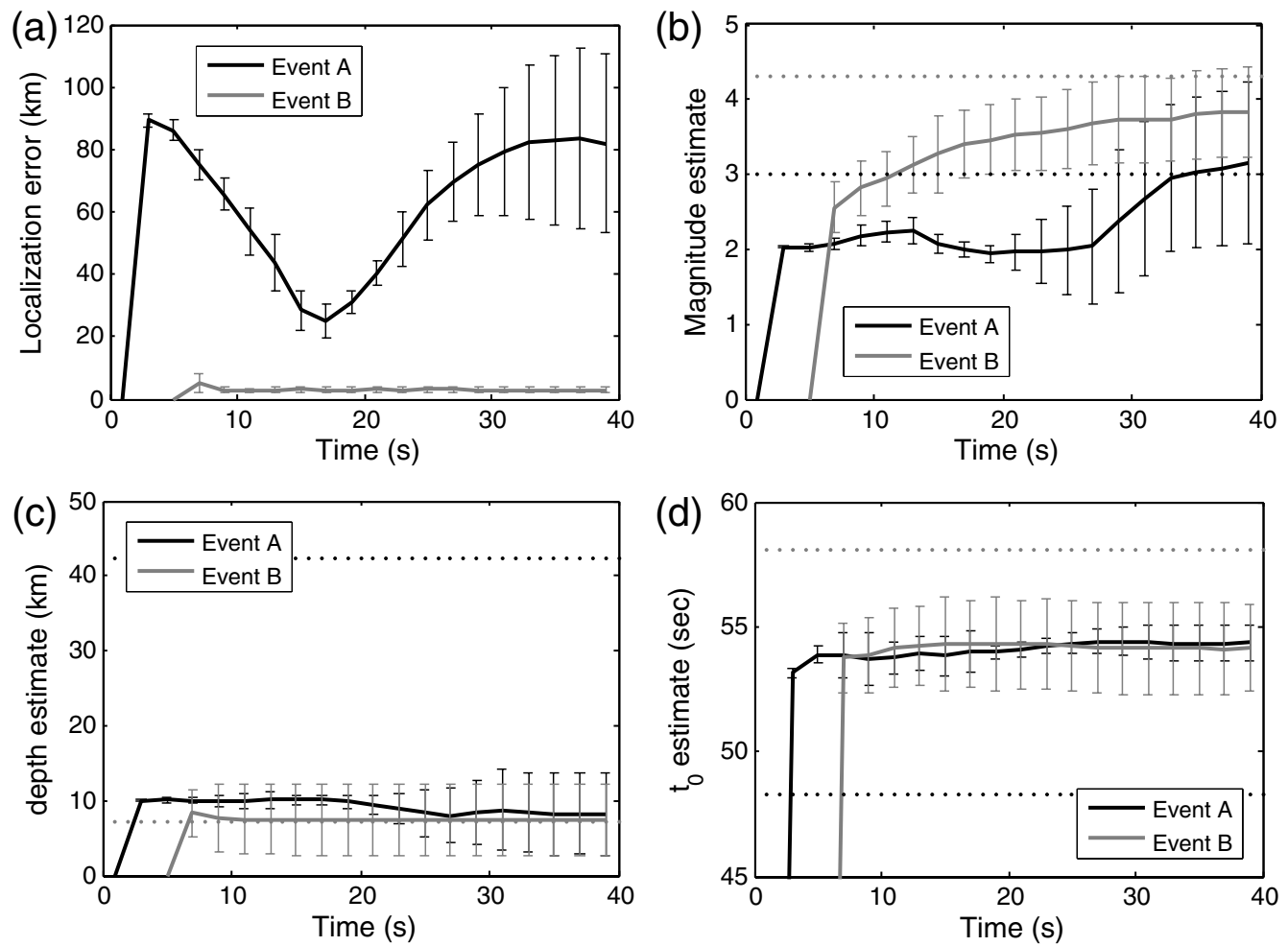

Figure 7. Results compiled from 20 independent runs in the period between 14:19:56 and 14:20:36 on 20 March 2011. The subfigures and included symbols are defined as in Figure 5. 
(a)

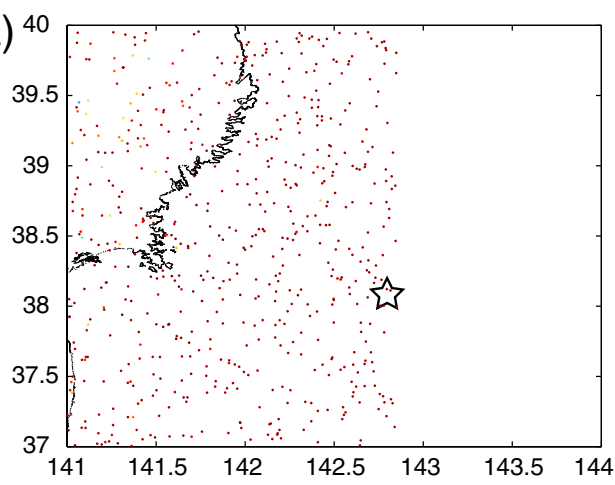

(c)

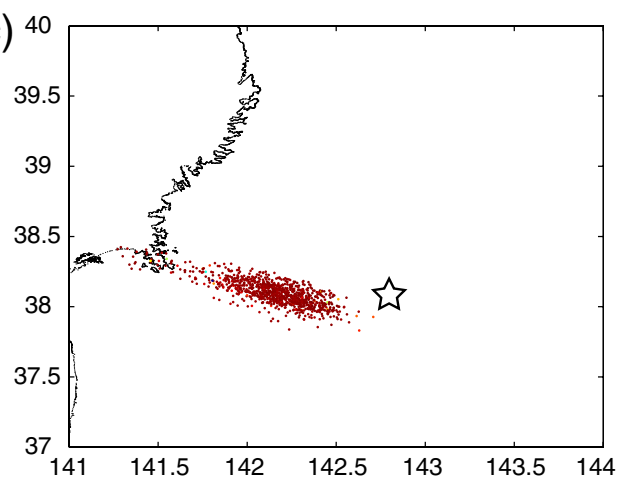

(e)

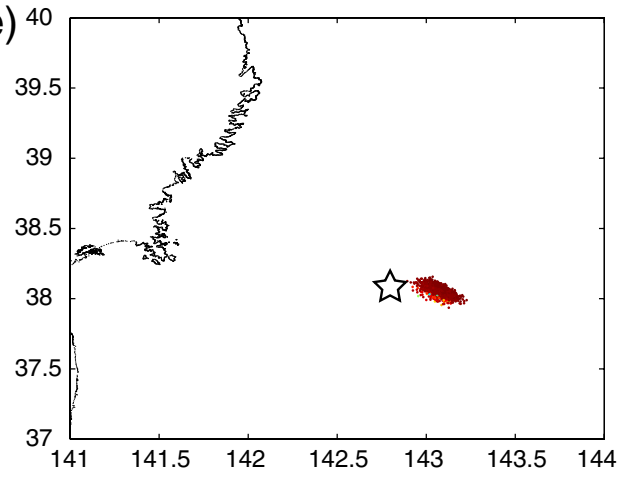

(b)

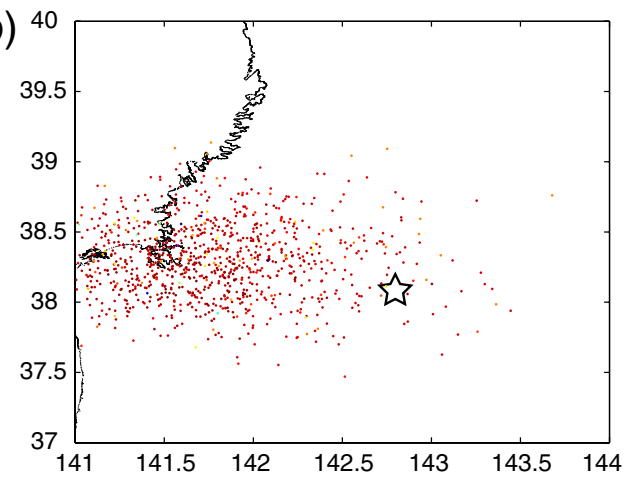

(d)

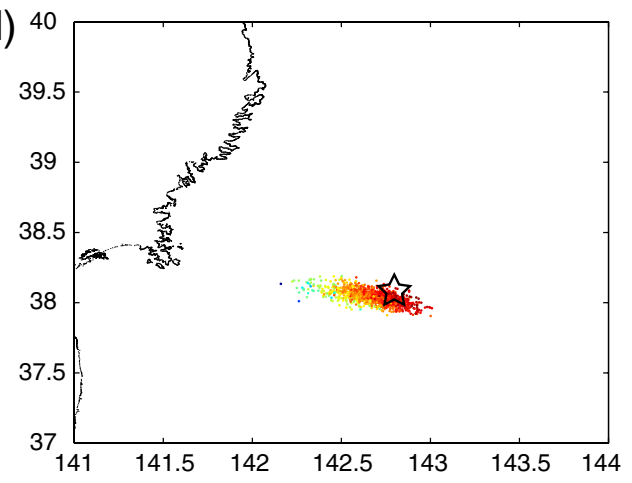

(f)

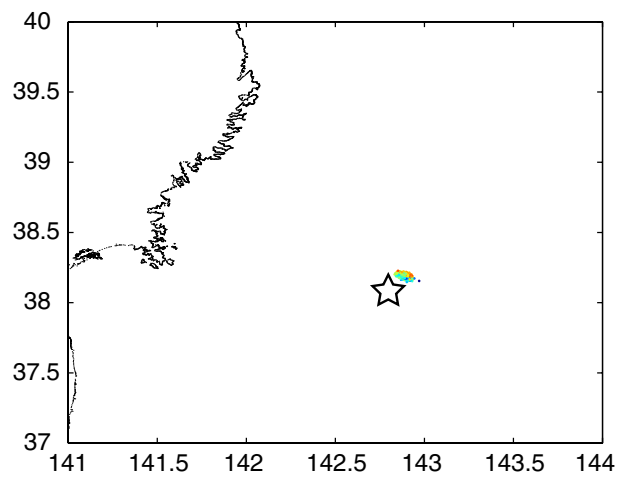

Figure 8. Distributions of 1000 particles visualized on the map at (a) $2 \mathrm{~s}$, (b) $7 \mathrm{~s}$, (c) $13 \mathrm{~s}$, (d) $22 \mathrm{~s}$, (e) $32 \mathrm{~s}$, and (f) $62 \mathrm{~s}$ after 14:46:46 on 11 March 2011. The symbols are defined as in Figure 4. The color version of this figure is available only in the electronic edition.

offshore earthquakes, the estimates converge in 20-30 s. In terms of localization error, we observed less than $20 \mathrm{~km}$ for inland earthquakes and $20-80 \mathrm{~km}$ for offshore events.

In order to classify multiple concurrent earthquakes, the use of nontriggered stations is important. The current JMA EEW system uses arrival times of waves at only the triggered stations in the hypocenter calculation. As a result, when multiple earthquakes occur around the same time and the later event occurs close to the wave arrival times of the earlier event, the EEW system treats these events as one single earthquake. If this is the case and the stations around the later event observe nonnegligible shakings, the current system may overestimate the magnitude because these stations are far away from the estimated hypocenter (i.e., the location of the earlier event). In our approach, the likelihood function uses information from not only the triggered stations but also the nontriggered ones. This design together with the adaptive measure of $A_{\text {noise }}$ allows the algorithm to identify unaffected regions between events and is therefore crucial in separating multiple concurrent earthquakes.

Another advantage of our approach is the use of regularized PF to circumvent the need for intensive computation that traditional grid search requires. Although a prior distribution is still required as mentioned in the Model section, such a distribution can be compiled from historical records. Alternatively, initial measurements can be used to select the appropriate priors to achieve better performance (Liu et al., 2011). This approach is also subject to several weaknesses. For example, the algorithm is sensitive to the choice of prior distribution, the number of particles, the values of $A_{\text {noise }}$, $\sigma_{\text {noise }}, \sigma_{P}$, and $\sigma_{S}$. Although these values can be adjusted and adapted in real time, it requires extensive empirical studies and analyses of historical records for the algorithm to be 

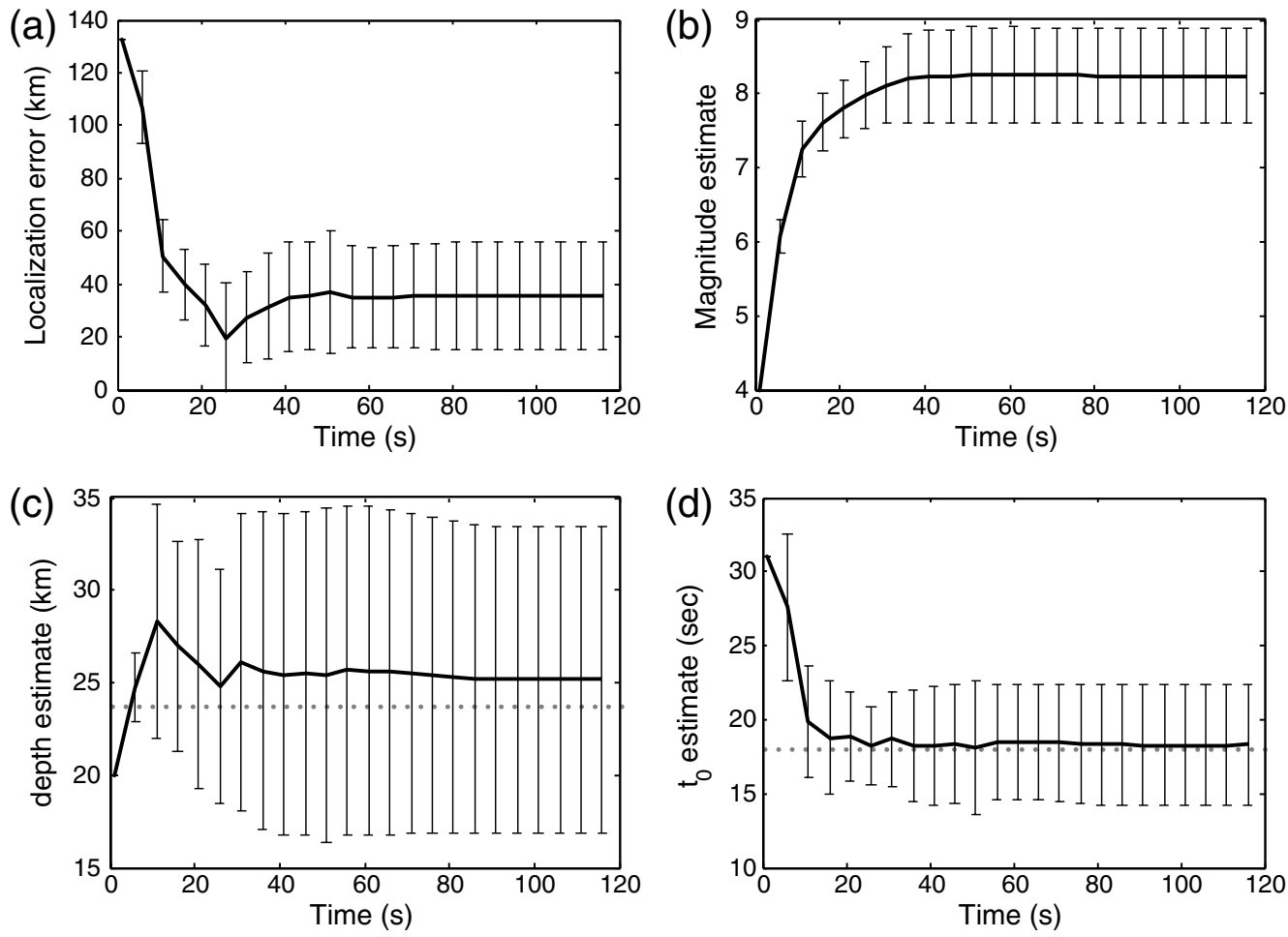

Figure 9. Results compiled from 15 independent runs for the period between 14:46:46 and 14:48:46 on 11 March 2011. The subfigures and included symbols are defined as in Figure 5.

robust. Some of the slow convergence and high variance results in the Results section may be attributed to suboptimal choices in these parameters.

In this paper, we use only three cases to test the proposed method, so we are currently carrying out more extensive evaluations of our method using many examples of multiple earthquake sequences that have occurred over the last several years.

As a side note, the performance of parameter estimation for multiple seismic events is limited by how well one can model the ground motion when multiple wavefronts overlap. In the algorithm proposed in the Model section (see Fig. A1), this model is not considered. Although the omission makes little difference in the case studies in which the events are spatially far apart (greater than $100 \mathrm{~km}$ ), if we want to apply the same technique to separate aftershocks from mainshock that occur close in time, then such model should be considered.

\section{Conclusion}

In the seismically active period, multiple earthquakes of similar or distant origins can take place at almost the same time. Failure to identify them as separate events leads to poor estimates of their parameters. The error in estimates can in turn cause false warnings. In this paper, we study the problem of detecting and classifying multiple earthquakes that occur close in time. Based on a Bayesian formulation that considers the possibility of having more than one event present at any given time, we propose a novel-likelihood function suitable for clas- sifying multiple concurrent earthquakes and present a sequential Monte Carlo heuristic whose complexity grows linearly with the number of events. The performance of the heuristic is empirically validated with three sets of JMA seismic records after the 2011 Tohoku earthquake. The initial studies show that the approach is able to successfully separate multiple events that occur close in space and time and estimate their parameters in real time to a reasonable degree of precision in comparison to official values determined by JMA in the postevent analyses. Although complete validation and characterization are required before this method applied in real-time detection, the initial results show that our approach can reduce the chance of overestimation of earthquake magnitude and, as a result, contribute to the design of a better EEW system.

\section{Data and Resources}

Waveform data used in the present study were extracted from continuous recordings of the stations within the Japan Meteorological Agency (JMA) strong-motion network. The JMA earthquake early warning (EEW) performance in three cases is available at http://www.data.jma.go.jp/svd/eew/ data/nc/pub_hist/2011/03/20110315013605/content/content _out.html, http://www.data.jma.go.jp/svd/eew/data/nc/pub _hist/2011/03/20110320141959/content/content_out.html, and http://www.data.jma.go.jp/svd/eew/data/nc/pub_hist/ 2011/03/20110311144640/content/content_out.html (last accessed July 2013). We use Seismic Analysis Code (http:// 
www.iris.washington.edu/software/sac/manual/fileformat .html, last accessed July 2013) for the data processing. The JMA attenuation relationship is available in the report of the second JMA EEW evaluation committee (http://www .data.jma.go.jp/svd/eqev/data/study-panel/eew-hyoka /t02/shiryou.pdf, last accessed July 2013).

\section{Acknowledgments}

The first author would like to thank Jim Mori at Kyoto University for the generous hosting and the warm welcome she received from all the group members. The authors would also like to thank Japan Meteorological Agency (JMA) for providing the strong-motion seismic data. This work was generously funded by the 2011 National Science Foundation East Asian and Pacific Summer Institute (EAPSI) Fellowship and the Funding Program for Next Generation World-Leading Researchers (NEXT Program).

\section{References}

Arulampalam, M., S. Maskell, N. Gordon, and T. Clapp (2002). A tutorial on particle filters for online nonlinear/non-Gaussian Bayesian tracking, IEEE Trans. Signal Process. 50, no. 2, 174-188.

Bickel, P. J., and E. Levina (2008). Regularized estimation of large covariance matrices, Ann. Stat. 36, 1-487.

Doucet, A., N. de Freitas, N. Gordon, and A. Smith (2001). Sequential Monte Carlo Methods in Practice, Statistics for Engineering and Information Science, Springer, New York, 159-175.

Durham, G., and J. Geweke (2013). Adaptive sequential posterior simulators for massively parallel computing environments, http://adsabs.harvard .edu/abs/2013arXiv1304.4334D (last accessed April 2014).

Hoshiba, M., and K. Iwakiri (2011). Initial 30 seconds of the 2011 Off the Pacific Coast of Tohoku earthquake ( $\left.M_{\mathrm{w}} 9.0\right)$-amplitude and tau(c) for magnitude estimation for earthquake early warning, Earth Planets Space 63, no. 7, 553-557.

Hoshiba, M., and T. Ozaki (2013). Earthquake early warning and tsunami warning of the Japan Meteorological Agency, and their performance in the 2011 Off the Pacific Coast of Tohoku earthquake $\left(M_{\mathrm{w}} 9.0\right)$, in Early Warning for Geological Disasters, F. Wenzel and J. Zschau (Editors), Springer-Verlag, Berlin, 1-28.

Hoshiba, M., K. Iwakiri, and N. Hayashimoto (2011). Outline of the 2011 Off the Pacific Coast of Tohoku earthquake $\left(M_{\mathrm{w}} 9.0\right)$-Seismicity: Foreshocks, mainshock, aftershocks, and induced activity, Earth Planets Space 63, no. 7, 513-518.

Japan Meteorological Agency (JMA) (2011). Earthquake early warning report for Tohoku 2011, http://www.jma.go.jp/jma/press/1104/28b/eew_hyouka_2.pdf (last accessed April 2014).

Katsumata, A. (2008). Recursive digital filter with frequency response of a mechanical seismograph, Technical Report, Seismology and Volcanology Research Department, Meteorological Research Institute, Tsukuba, Japan.

Liu, A. H., J. Bunn, and K. M. Chandy (2011). Sensor networks for the detection and tracking of radiation and other threats in cities, in The 10th International Conference on Information Processing in Sensor Networks (IPSN), Chicago, Illinois, 12-14 April 2011, 1-12.

Liu, J. S., and R. Chen (1998). Sequential Monte Carlo methods for dynamic systems, J. Am. Stat. Assoc. 93, 1032-1044.

Miao, L., J. J. Zhang, C. Chakrabarti, and A. Papandreou-Suppappola (2010). A new parallel implementation for particle filters and its application to adaptive waveform design, 2010 IEEE Workshop on Signal Processing Systems (SIPS), 19-24.

Musso, C., N. Oudjane, and F. Legland (2001). Improving regularized particle filters, in Sequential Monte Carlo Methods in Practice, A. Doucet, N. de Freitas, and N. Gordon (Editors), Vol. 12, Statistics for Engineering and Information Science, New York, 247-271.

Sagiya, T., H. Kanamori, Y. Yagi, M. Yamada, and J. Mori (2011). Rebuilding seismology, Nature 473, 146-148.
Ueno, H., S. Hatakeyama, T. Aketagawa, J. Funasaki, and N. Hamada (2002). Improvement of hypocenter determination procedures in the Japan Meteorological Agency, Q. J. Seismol. 65, nos. 1/4, 123-134.

\section{Appendix}

The algorithm in Figure A1 describes the outline of regularized particle filter for multiple seismic event detection. The "CONVERGED" criteria can be substituted with desired conditions (e.g., change in estimates $\left\|\hat{\theta}_{t-10}-\hat{\theta}_{t-1}\right\|<\delta$ ).

\section{Computer Science}

California Institute of Technology

1200 E California Blvd

Pasadena, California 91125

aliu@cms.caltech.edu

(A.L.)

Disaster Prevention Research Institute

Kyoto University

Gokasho, Uji 611-0011, Japan

masumi@eqh.dpri.kyoto-u.ac.jp

(M.Y.)

Manuscript received 2 August 2013;

Published Online 13 May 2014

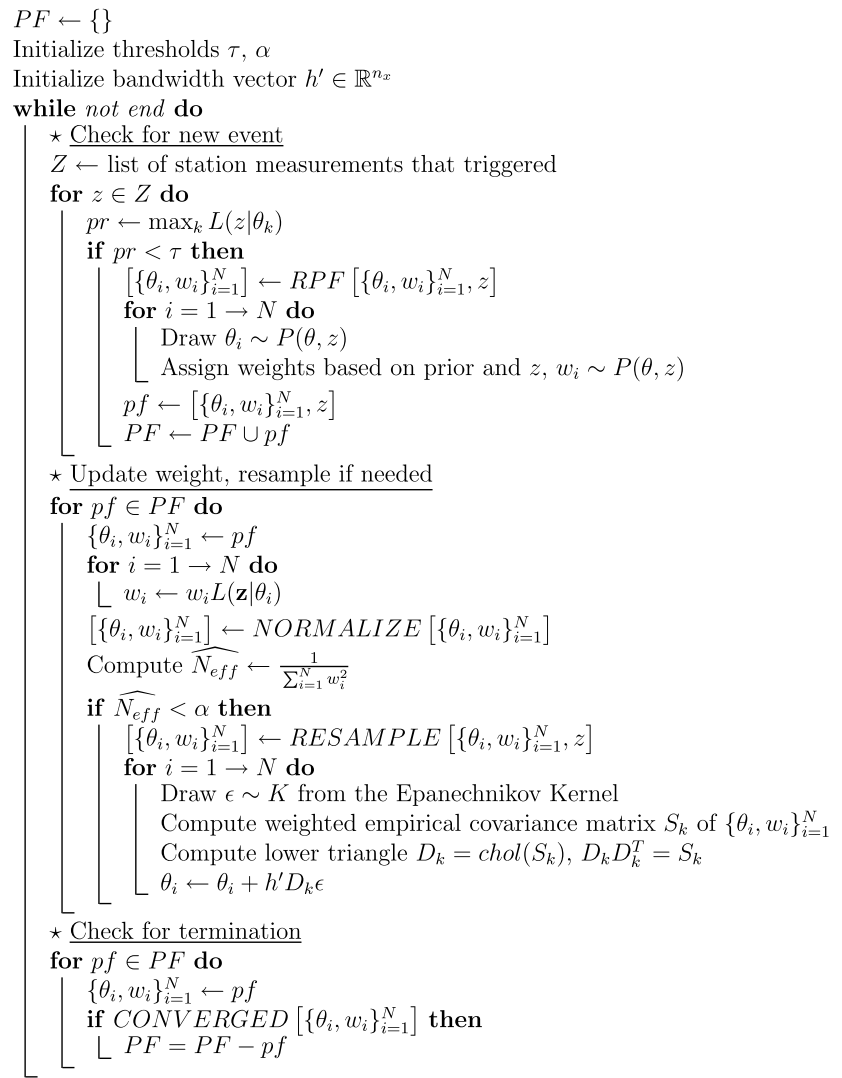

Figure A1. Pseudocode for particle filter implementation of Bayesian parameter estimation system for multiple events. 